\title{
Meta-analysis: compared with anti-CCP and rheumatoid factor, could anti-MCV be the next biomarker in the rheumatoid arthritis classification criteria?
}

https://doi.org/10.1515/cclm-2019-0167

Received February 11, 2019; accepted April 1, 2019; previously published online May 29, 2019

\section{Abstract}

Background: Previous reviews of the diagnosis for rheumatoid arthritis (RA) have not compared anti-mutated citrullinated vimentin (MCV) with anti-cyclic citrullinated peptide (CCP) and rheumatoid factor (RF) in respect of sensitivity, specificity and the area under the curve (AUC) against disease controls for differential diagnosis. This meta-analysis aims to evaluate the value of anti-MCV in the diagnosis for RA, the combined sensitivity of anti-MCV and anti-CCP, and certain clinical characteristics related to the performance of anti-MCV.

Methods: Medline, Embase, Cochrane Library and Web of Science were searched for articles published up to 25 August 2018. A total of 33 studies including 6044 RA patients and 5094 healthy or disease controls achieved inclusive criteria. QUADAS-2 was applied to evaluate the quality of the included studies. The bivariate random effects model was employed in primary data synthesis to evaluate the diagnostic performance.

Results: The sensitivity of anti-MCV, anti-CCP and RF in RA diagnosis against a disease control group was $0.71,0.71$, 0.77 , with the specificity of $0.89,0.95,0.73$, and the AUC of the SROC of $0.89,0.95,0.82$, respectively. The predesign of the primary study and diagnostic criteria were statistically significant as sources of heterogeneity. Anti-MCV and anti-CCP tests demonstrated a sensitivity of 0.77 when performed in parallel, with a sensitivity of 0.60 when performed in series; whereas, the combination of anti-MCV and RF presented a sensitivity of 0.64 when used in series.

ajia-Ning Zhu, Liu-Yan Nie and Xiao-Yong Lu contributed equally to this work.

*Corresponding author: Hua-Xiang Wu, MD, PhD, Department of Rheumatology, Second Affiliated Hospital, School of Medicine, Zhejiang University, No. 88 Jiefang Road, Hangzhou 310009, P.R. China, Phone/Fax: +86-571-89713754, E-mail: wuhx8855@zju.edu.cn Jia-Ning Zhu, Liu-Yan Nie and Xiao-Yong Lu: Department of Rheumatology, Second Affiliated Hospital, School of Medicine, Zhejiang University, Hangzhou, P.R. China
Conclusions: Anti-MCV demonstrates comparable diagnostic value to anti-CCP and RF, thus it can be an effective diagnostic marker for RA and may be written into the next authoritative criteria.

Keywords: anti-cyclic citrullinated peptide antibodies; anti-mutated citrullinated vimentin antibodies; diagnosis; rheumatoid arthritis; rheumatoid factor.

\section{Introduction}

Rheumatoid arthritis (RA) is one of the most prevalent chronic inflammatory diseases, with an incidence of $0.5 \%-1.0 \%$ worldwide [1]. It was estimated to be present in $2.4 \%$ of the population aged $>65$ years, with the prevalence appearing to increase with age [2]. The severe outcome of destructive and deforming arthritis influences impairment of functional capacity and results in low quality of life in patients with RA [3].

Poor as the prognosis of patients with RA is, early diagnosis is regarded as the key role for improvement, and high efficacy is seen with this strategy in reducing joint destruction, retarding radiologic progression and decreasing functional disability [4]. Anti-citrullinated peptide antibodies (ACPAs) and rheumatoid factor (RF) belong to the serum biomarkers involved in the 2010 American College of Rheumatology (ACR)/the European League Against Rheumatism (EULAR) RA classification criteria, no doubt underscoring the serodiagnostic utility of these autoantibodies in RA. Furthermore, ACPAs include anticyclic citrullinated peptide (anti-CCP), anti-mutated citrullinated vimentin (anti-MCV), anti-centriolin antibody (anti-CEP-1) and so on, among which anti-CCP is widely used [5].

To date, three published meta-analysis have already tested which is better for diagnosis of RA between antiMCV and anti-CCP [6-8]. Nevertheless, there is a lack of information on studies concluding the comparison of anti-MCV and anti-CCP with respect to diagnostic accuracy in RA. Restricted by the limited numbers of studies which fulfill the inclusive criteria, these reviews did not 
discuss the specific area of diagnostic accuracy compared to anti-CCP, and it was suggested that anti-MCV could be tested in patients suspected of RA who were seronegative for anti-CCP and RF. Based on previous studies, we decided to evaluate how anti-MCV performs in the diagnosis of RA by increasing the number of included studies and exploring the sensitivity and specificity of anti-MCV, anti-CCP and RF in the diagnosis of RA. Moreover, the efficacy of anti-MCV combined with anti-CCP, and the impact of diagnosis at different cutoff values for anti-MCV were summarized, and receiver operator characteristic curve (ROC) of anti-MCV, anti-CCP and RF were evaluated to identify the best cutoff for diagnosis. In addition, results of studies that assessed the clinical heterogeneity of index testing methods, the gold standard applied for the diagnosis, index cutoff values and the predesign of the primary studies are discussed [8].

\section{Materials and methods}

\section{Search strategies}

This meta-analysis was performed following the guidelines of the Cochrane Handbook for Systematic Reviews of Diagnostic Test Accuracy. Four databases were searched for this study: Medline, Embase, Cochrane Library and Web of Science, with publication time up to 25 August 2018. A combination of MeSH terms and keywords pertaining to RA ("Arthritis, Rheumatoid" OR "rheumatoid arthr" OR "RA"), AND anti-MCV ("MCV” OR "mutated citrullinated vimentin") were applied to the search strategies. The reference lists of all the included studies were searched to identify any additional eligible studies.

\section{Study selection}

Titles and abstracts were independently screened by two reviewers (Zhu and Nie). Full articles were retrieved and reviewed for the following inclusion criteria:

1. studies were prospective or retrospective or crosssectional study of the diagnostic value of MCV, with or without CCP and/or RF in RA study with human subjects;

2. studies included a healthy and/or other diseases control groups for comparison;

3. RA patients fulfilled either the 1987 ACR classification criteria or the 2010 ACR/EULAR classification criteria, as which have been approved by the professional and scientific authorities;
4. studies evaluated MCV, with or without CCP and/or $\mathrm{RF}$ levels in serum, and were quantitative tests;

5. studies provided the necessary data, e.g. the number of true positive, true negative, false positive, and false negative, sensitivity, specificity, or likelihood ratios, to construct $2 \times 2$ contingency tables of levels of MCV, with or without CCP and/or RF for the diagnosis of RA.

Studies were excluded if they were not available in English or included duplicate data. No age restriction on participants was applied here.

\section{Data extraction}

Two reviewers ( $\mathrm{hu}$ and $\mathrm{Nie}$ ) individually extracted the data, with discrepancies resolved by discussion. When it was difficult to reach an agreement, a third reviewer $(\mathrm{Lu})$ was consulted. When results of different cutoff values were reported on an index test, data from the recommended cutoff value according to the manufacturers' instructions were extracted. Where there was more than one type of assay for the same index test, the more frequently used one among included studies was extracted. Where more than one subtype of the same kind of index test was available from the study, the more widely used one was included for the review, to be more specific, anti-CCP2 IgG and RF IgM were chosen as the representation.

\section{Quality assessment}

The quality of each included study was independently assessed by the two reviewers (Zhu and Nie), using the revised version of the Quality Assessment of Diagnostic Accuracy Studies (QUADAS-2) tool. Any disagreements were resolved through discussion.

\section{Statistical analysis}

Data analysis was performed using Stata 12 software (Midas commands) (StataCorp LP, College Station, TX, USA), Revman 5.2 (The Nordic Cochrane Centre, The Cochrane Collaboration, Copenhagen, Denmark) and $\mathrm{R}$ version 3.5.0 (R Foundation for Statistical Computing, Vienna, Austria).

The bivariate random effects model based on the binomial distribution for sensitivity and specificity 
was employed in primary data synthesis, with inferences made about summary estimates of sensitivity and specificity and their 95\% confidence intervals (CIs). Derived logit estimates of sensitivity, specificity based on parameters estimated by the bivariate model were used to plot a hierarchical summary ROC curve, with a summary point estimate, $95 \%$ confidence region and $95 \%$ prediction region presented. The area under the curve (AUC) is the average TPR over the entire range of FPR values.

$\mathrm{I}^{2}$ is viewed as the index to assess the heterogeneity, which calculates the percentage of total variation across studies caused by heterogeneity rather than chance. $\mathrm{I}^{2}$ ranges from $0 \%$ to $100 \%$, with $0 \%$ indicating no observed heterogeneity, and values greater than $50 \%$ suggesting substantial heterogeneity. Covariates regarding any test performance that may contribute to the heterogeneity were introduced into a regression as the dependent variable.

To investigate publication bias, the funnel plot with a superimposed regression line of diagnostic log odds ratio against 1 /sqrt (effective sample size), weighting by effective sample size was conducted, and $\mathrm{p}<0.10$ for the slope coefficient suggests significant asymmetry.

\section{Results}

\section{Search results}

The initial search identified 704 articles, of which 300 were duplicates and 302 were excluded according to the title and abstract (Figure 1). One hundred and two fulltext articles were reviewed, of which 25 were excluded as we were unable to extract sufficient information to construct $2 \times 2$ tables, 15 were excluded as they did not include a control group, seven were excluded due to a lack of a golden diagnostic tool, specifically, neither the 1987 ACR classification criteria nor the 2010 ACR/EULAR classification criteria for RA was adopted in these studies, 15 were conference abstracts, two were systematic reviews, two were duplicate reports and another two were not available in English. Therefore, the meta-analysis was conducted based on the remaining 33 studies [9-41] (Table 1).

\section{Methodological quality of included studies}

QUADAS-2 was selected as the guide for methodological quality evaluation for the included studies, this evaluates
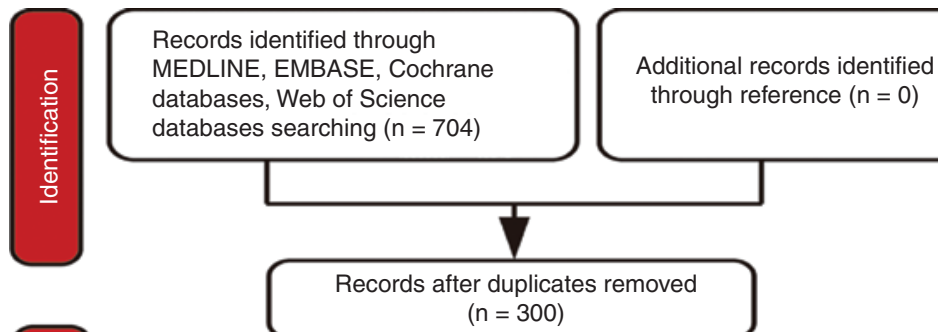

Records after duplicates removed $(n=300)$
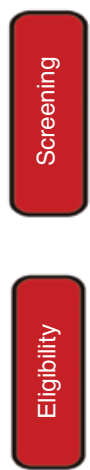

Figure 1: Flowchart of selection of studies for inclusion in the meta-analysis. 


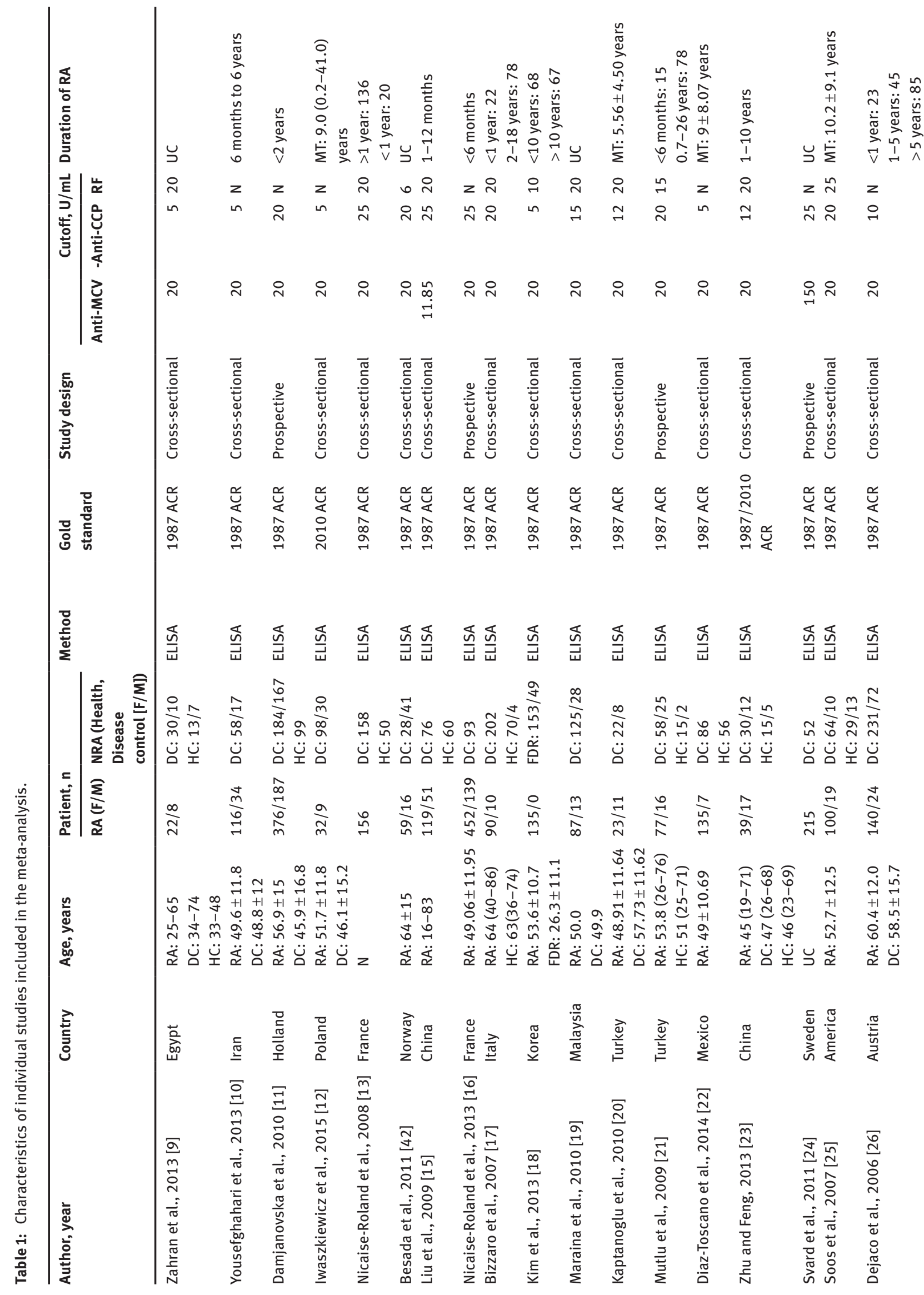




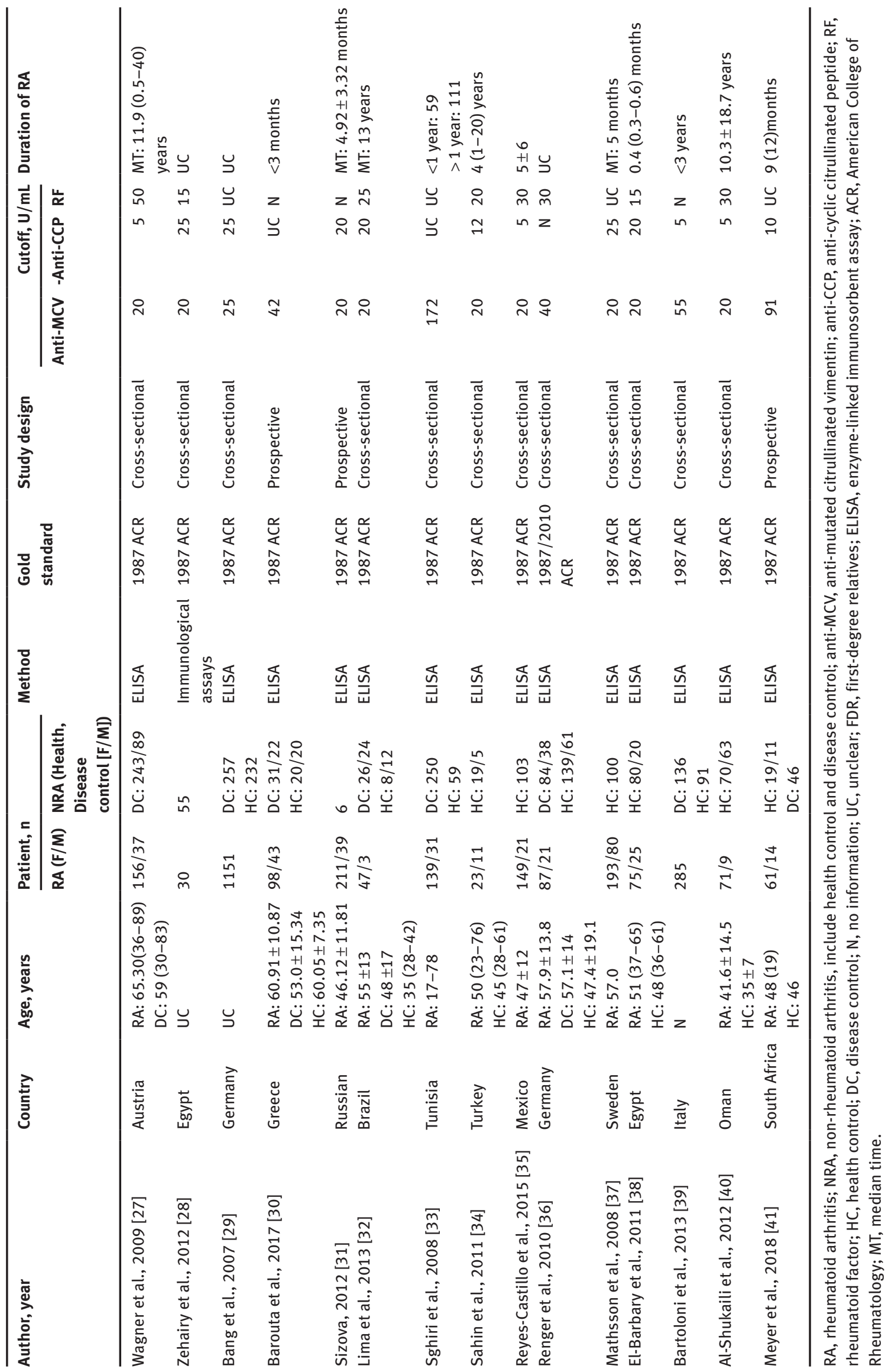


the risk of bias of each study cohort on four categories (patient selection, index test, reference standards, flow and timing), and the concerns regarding applicability on three categories (patient selection, index test, reference standards). The risk was graded as high, low or unclear, and an overall risk grades for each category mentioned were estimated (Figure 2). About half of the studies showed

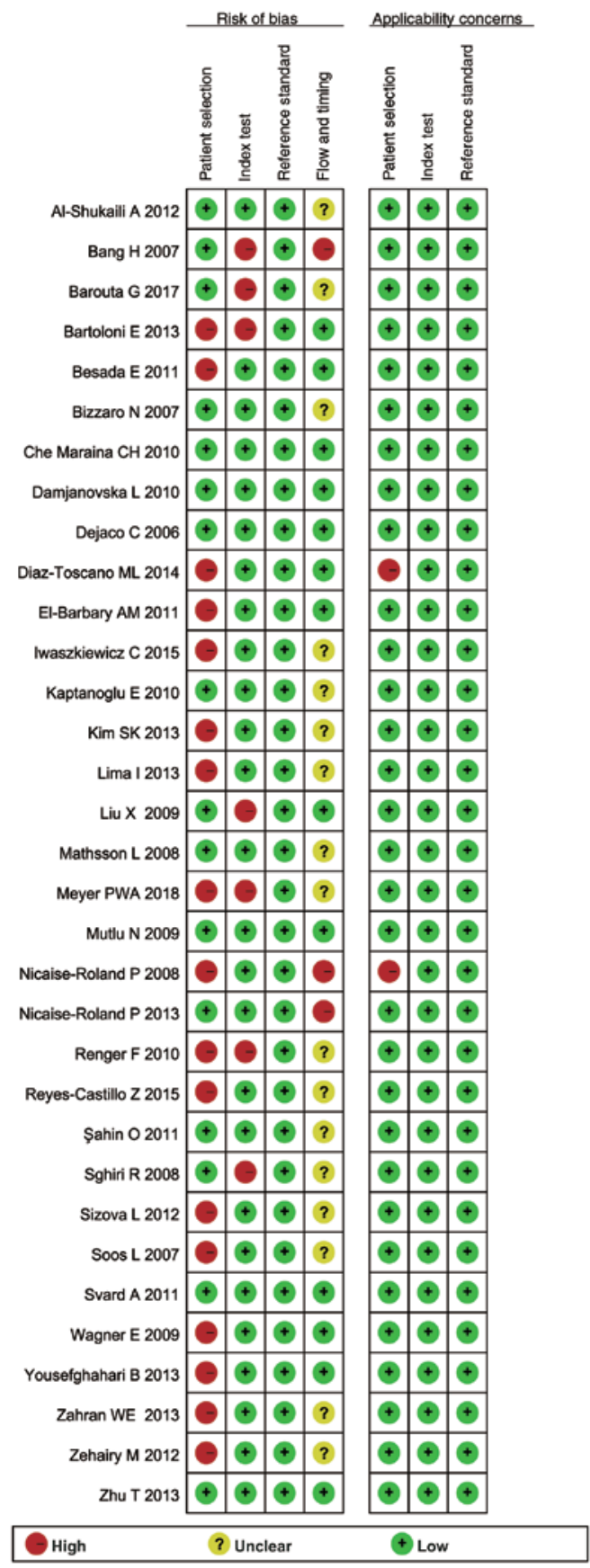

Figure 2: Risk of bias and applicability concerns summary. Each item in the graph was described with + (low risk of bias), - (high risk of bias) and ? (unclear). a high risk for patient selection because a case-control design was often difficult to avoid and a consecutive or random sample of cohorts was difficult to satisfy (see Supplementary material, Figure 1). Around $50 \%$ of the studies presented an unclear risk for flow and timing, based on the fact that except for the gold standard of diagnosis for RA, the reference standards for other rheumatic diseases were unclear, therefore making it challenging to decide whether the disease control groups received the same gold standard. Nearly all the studies demonstrated a low risk among all three categories of the concerns regarding applicability.

\section{Diagnostic value of anti-MCV with RA}

The diagnostic value of MCV to RA vs. the disease control group mainly consisted of other rheumatic diseases, some infectious diseases with similar clinical features to RA, one study with unaffected first-degree relatives from RA families is also reported here. Among the 33 studies included in the present meta-analysis, eight of which provided information only on healthy controls rather than disease controls. According to the forest plot, the remaining 25 included studies [9-13, 15-30, 32, 41, 42] showed a combined sensitivity of 0.71 [0.64-0.77] ( $95 \% \mathrm{CI})$, specificity of 0.89 [0.85-0.92] (Figure 3). The SROC curve showed an AUC of 0.89 [0.86-0.91] (Figure 4).

The $I^{2}$ of combined sensitivity was 94.73 [93.42-96.03], with $\mathrm{p}=0.00$ for $\mathrm{Q}$ test; similarly, the $\mathrm{I}^{2}$ of combined specificity was 88.32 [84.66-91.98], with $\mathrm{p}=0.00$ for Q test. A meta-regression was performed due to the obvious heterogeneity, with covariates including testing methods of MCV with its cutoff value, disease duration, diagnostic criteria and predesign of the primary study. As a result, the predesign of the primary study presents as a significant contributor to the heterogeneity for both sensitivity and specificity, with $\mathrm{p}=0.00$ and $\mathrm{p}=0.01$ respectively, while diagnostic criteria contribute to the heterogeneity for specificity $(\mathrm{p}=0.05)$.

Publication bias was examined using the Deeks' funnel plot asymmetry test, symmetry in the data and a low likelihood of publication bias are observed in the present study $(\mathrm{p}=0.20)$.

\section{Clinical subgroup analysis}

The predesign of the study and the diagnostic criteria were observed to be statistically significant as sources of heterogeneity by meta-regression among the 25 studies where 

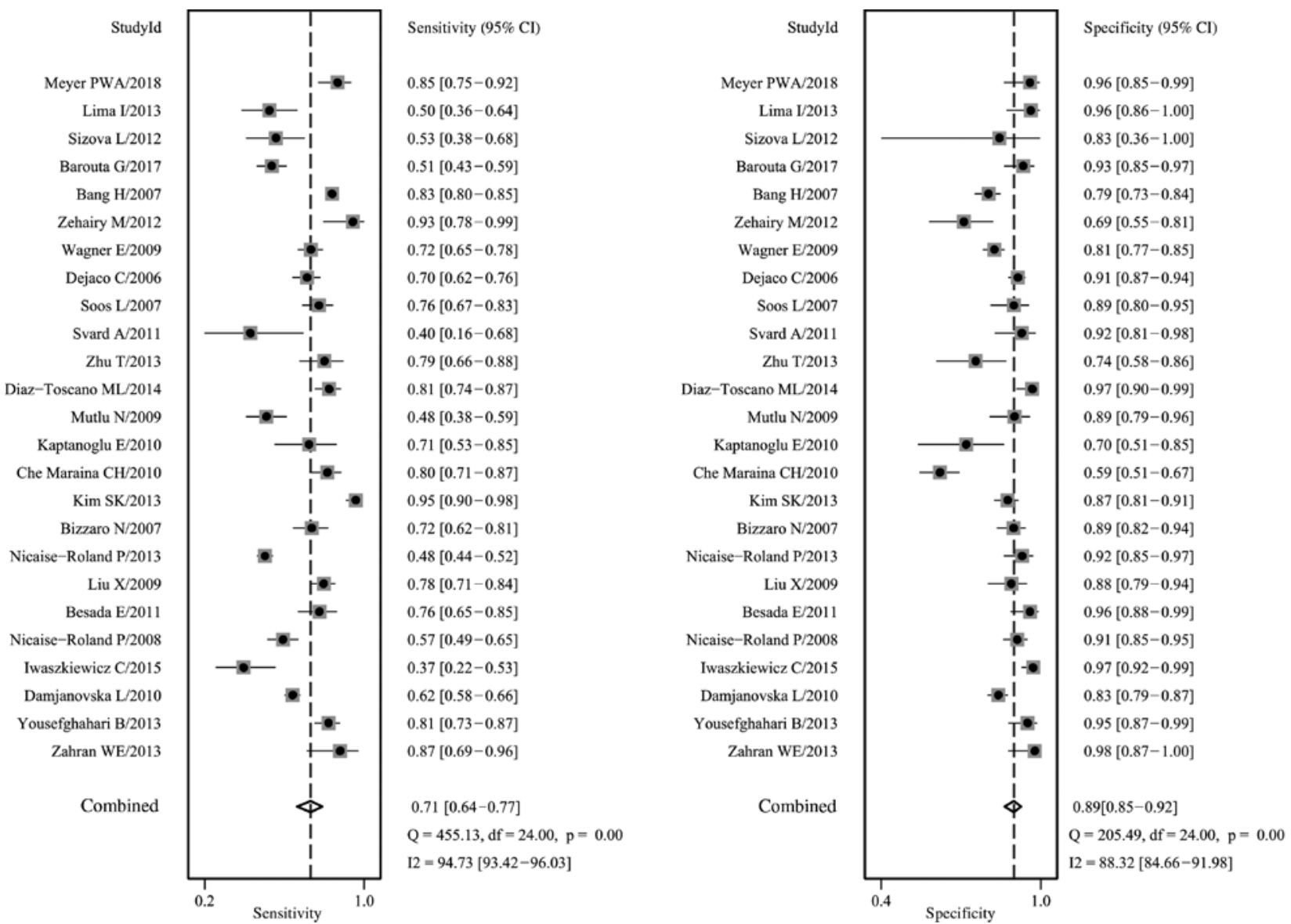

Figure 3: Forest plot of coupled sensitivity and specificity for anti-MCV in the diagnosis of RA vs. DC.

The point estimate of sensitivity and specificity of each study is shown as a solid square on a line that represents the $\mathrm{Cl}$. The continuous vertical line represents the weighted average sensitivity and specificity and dotted vertical lines represent the $\mathrm{Cl}$.

the information on the disease control group was available, therefore, these two covariates were investigated by subgroup analysis. Clinical subgroups of different testing

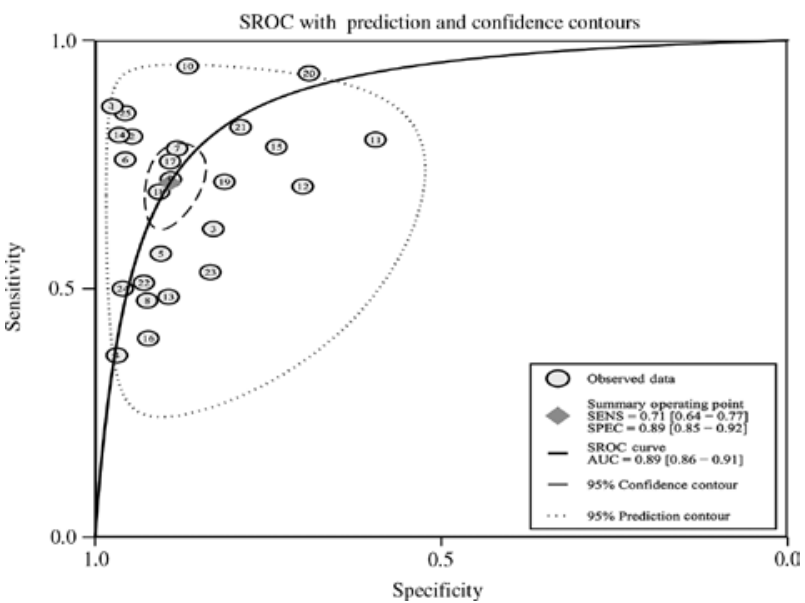

Figure 4: SROC curve for anti-MCV in diagnosis of RA vs. DC. Each circle represents a study and the asterisk represents the global estimate. methods of MCV with its cutoff value and disease duration were also analyzed, because of their clinical importance in RA diagnosis.

Seven studies $[11,16,21,24,30,31,41]$ with a prospective study predesign were distributed into one subgroup. The results of this subgroup suggested that according to the forest plot, the combined sensitivity was 0.57 [0.46$0.68]$, the specificity was 0.90 [0.86-0.94] (see Supplementary material, Figure 2). Among those 25 studies, only Iwaszkiewicz et al. [12] used the ACR/EULAR 2010 criteria as its diagnostic criteria for RA, while the rest adopted ACR 1987. Nineteen studies that used an enzyme-linked immunosorbent assay (ELISA) as its testing method for $\mathrm{MCV}$, with the cutoff set to $20 \mathrm{U} / \mathrm{mL}$ were assigned to one subgroup. The subgroup of uniform cutoff demonstrated a combined sensitivity of 0.70 [0.62-0.77], specificity of 0.90 [0.85-0.93] according to the forest plot (see Supplementary material, Figure 3 ).

Overall, the subgroup of the prospective study predesign demonstrated the lowest heterogeneity for both sensitivity and specificity and compared with all the 
other results in the present review, the value of $\mathrm{I}^{2}$ can be reduced by up to $10 \%$ for sensitivity, and $30 \%$ for specificity. The heterogeneity of the combined sensitivity among these two subgroups ranged from $81.56 \%$ to $93.69 \%$, represented by $\mathrm{I}^{2}$; the $\mathrm{I}^{2}$ of specificity ranged from $57.47 \%$ to $89.92 \%$, and $Q$ test suggested $p=0.00$, except for the specificity of the prospective study predesign subgroup $(\mathrm{p}=0.04)$.

\section{Comparison between MCV, CCP and RF}

Among the 33 studies included for the present metaanalysis, the results of anti-CCP tests from the disease control group were not available for nine of them [11, 29, $31,33-38]$. The remaining 24 included studies [9-28, 30, $32,39,41,42]$ which showed a combined sensitivity of 0.71 [0.64-0.77] and a specificity of 0.95 [0.94-0.97] (Figure 5) for anti-CCP according to the forest plot. The SROC curve showed an AUC of 0.95 [0.92-0.96] (see Supplementary material, Figure 4). With regard to RF, among the 33 originally included studies, 17 [10-12, 14, 16, 22, 24, 27, 29, 31, 34-40] of which lacked information of RF tests from the disease controls, only about half of the studies could be used to perform the following analysis. The result demonstrated a combined sensitivity of 0.77 [0.68-0.84], a specificity of 0.73 [0.63-0.82] for RF and an AUC of 0.82 [0.78-0.85] according to SROC curve (see Supplementary material, Figures 5 and 6). However, the results revealed substantial heterogeneity among the studies for each diagnostic marker. The $\mathrm{I}^{2}$ of sensitivity for CCP was 92.70 [90.67-94.74], and that of specificity was 79.93 [72.4487.42]. A lower heterogeneity of 89.94 [86.13-93.75] was observed in sensitivity for RF, with $\mathrm{I}^{2}$ of specificity being 93.37 [91.55-95.59]. The $\mathrm{p}$-value for the $\mathrm{Q}$ test was 0.00 for all the four heterogeneity results mentioned here.
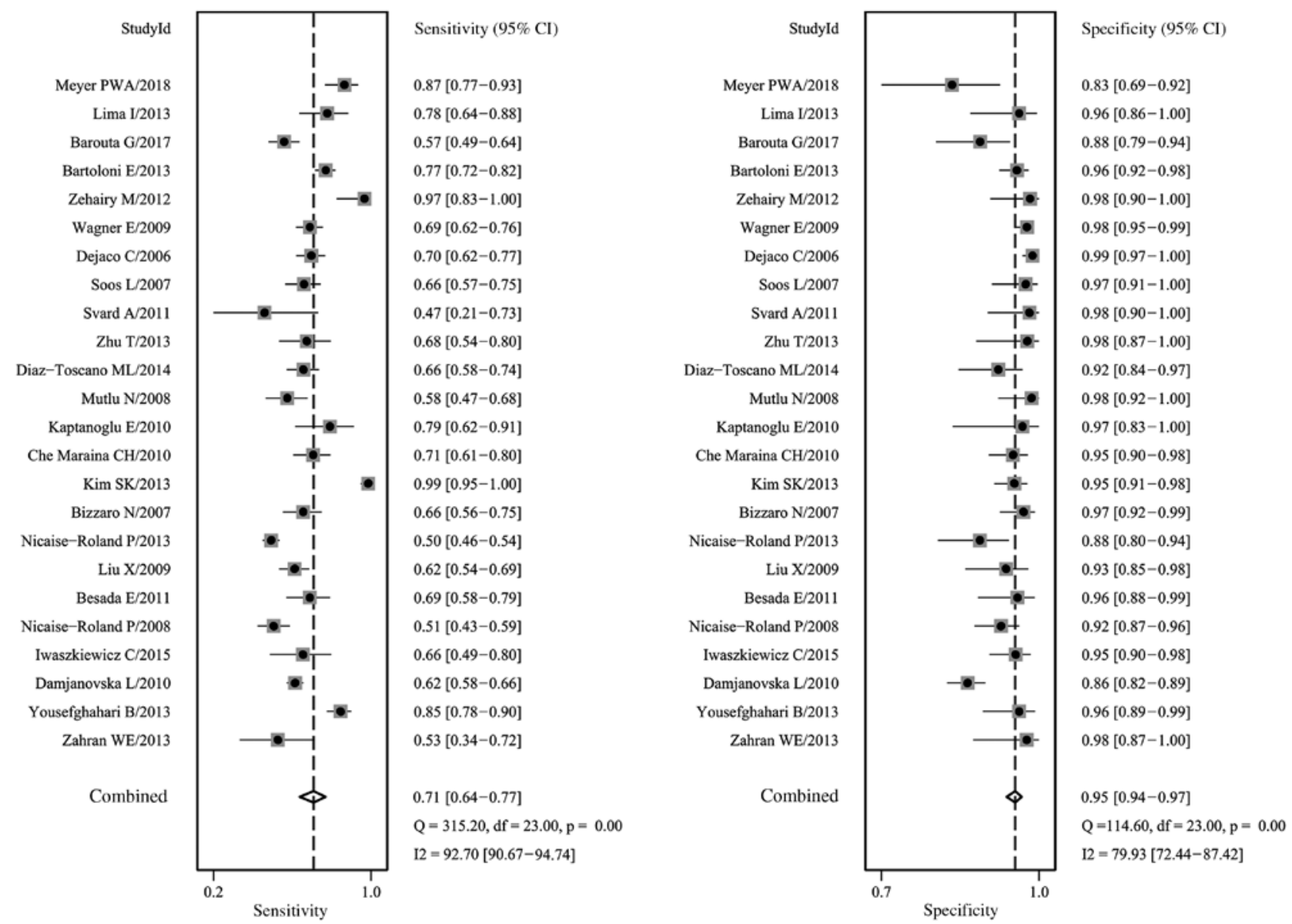

Figure 5: Forest plot of coupled sensitivity and specificity for anti-CCP in the diagnosis of RA vs. DC.

Forest plots (for sensitivity and specificity) and pooled estimates $\left(95 \% \mathrm{Cl}\right.$ ) by using the $\mathrm{I}^{2}$ test (quantified by $\mathrm{p}$-value $\left.<0.01\right)$ were yielded to show heterogeneity between studies. 


\section{Combinations of markers in series and in parallel}

Ten studies [13-15, 22-25, 37-40] were included to calculate the combined sensitivity of anti-MCV and anti-CCP when performed in parallel. The result suggested a sensitivity of 0.77 [0.72-0.82], with $\mathrm{I}^{2}=80.1 \%$ [64.1\%-88.9\%]. Eleven studies $[13,15,22,24,25,30,35,37-40]$ contained information on the performance of anti-MCV and anti-CCP in series and their combined sensitivity was 0.60 [0.540.67], with $\mathrm{I}^{2}=84.7 \%$ [74.8\%-90.7\%]. Nevertheless, no information on the control groups can be abstracted from almost all the studies, so the combined specificity was not available. Three studies $[32,36,37]$ were incorporated to evaluate the serial tests for anti-MCV and RF, with the combined sensitivity of 0.64 [0.40-0.83], with $\mathrm{I}^{2}=95.7 \%$ [90.7\%-98.0\%]. All these calculations regarding the diagnostic values of combinations of the markers were done using random effects models, and all Q tests suggested $\mathrm{p}<0.0001$.

\section{Discussion}

ACPAs play a crucial role in the differential diagnosis of patients with joint swelling and arthralgia, as well as prognosis evaluation and tailored therapy for RA [43]. As one of the members of the ACPA family, anti-Sa antibodies were first described in the serum of RA patients in 1994. Citrullinated forms of vimentin were recognized as the major antigen of Sa in 2004. MCV was discovered in 2007 to determine the existence of additional vimentin modifications or different isoforms of vimentin in order to improve the diagnostic efficiency of RA [4, 5, 44]. Recently, anti-MCV IgG isotype autoantibodies have been quantitatively measured by ELISA assay mostly for the diagnosis of RA, showing high sensitivity and specificity [44, 45]. It is known that the citrulline-driven immunological response is specifically associated with RA. The formation of autoantibodies against citrullinated vimentin interacts with the osteoclast lineage, thus promoting bone loss and leading to joint destruction $[46,47]$. Here, we perform a meta-analysis to identify the diagnostic value of anti-MCV for RA again, in order to compensate for unsolved limitations.

According to the already published reviews, the sensitivity of anti-MCV was reported as $0.69-0.77$ with the specificity against either healthy controls or disease controls to be from 0.89 to 0.94 , and an AUC of 0.88 was observed against healthy controls in one meta-analysis, which was consistent with our meta-analysis. In consideration that healthy controls may not be the comparator group in a clinic setting, in the present review we restricted the studies by RA diagnosis vs. disease control group only, and according to the AUCs, we revealed that anti-CCP performed the best diagnostic value, followed by anti-MCV and RF.

Individual markers were combined in parallel and in series to improve sensitivities and specificities in this review, and it is the common practice in clinical diagnosis. In total, there were four different kinds of combinations in parallel and four in series in the combinations of anti$\mathrm{MCV}$, anti-CCP and RF. Unfortunately, due to the lack of studies containing information about combined test characteristics, no AUCs can be used to infer a best combination of markers for RA diagnosis.

The heterogeneity of both sensitivity and specificity in studies of RA diagnosis against a disease control group remained high across all three diagnostic markers. Among the studies included in this review, ELISA, with the kit produced by Orgentec Diagnostika $\mathrm{GmbH}$ (Germany), recommended the cutoff value at 20 $\mathrm{U} / \mathrm{mL}$; while the POCT LFIA device and other immunological assays were also used, with cutoff set at different values. ELISAs were mainly used to detect anti-CCP2 IgG, however, either the second or the third generation anti-CCP ELISA was popularly used among the studies, causing variable cutoff values ranging between 5 and 25 $\mathrm{U} / \mathrm{mL}$. Nephelometry was a popular method to test RF, followed by ELISA which was less popular. Although sometimes the positivity of IgG, IgA and IgM RF were all available, IgM RF was chosen to represent the diagnostic value of RF because of its greater universality; cutoffs of which ranges from 6 to $30 \mathrm{U} / \mathrm{mL}$. Therefore, the high heterogeneity can be partially explained by different kinds of specific testing methods and reagents, as well as the different cutoffs set by individual researchers, which varied in different laboratories or institutions and across time. In addition, certain baseline clinical characteristics of RA patients may probably contribute to the heterogeneity, such as baseline inflammation indicators, including the erythrocyte sedimentation rate and C-reactive protein, DAS28, the general assessment score of RA, current antirheumatic therapy, including the usage and dosage of DMARDs and corticosteroids, radiography evaluation, like the Larsen score, and so on. However, this information could only be extracted from a limited small number of studies.

One of the main prognostic factors for RA is bone erosion, which results in the severe outcome of destructive and deforming arthritis. However, only six [12, 15, 27, $30,31,37$ ] of the included studies in the present review discussed the relation of anti-MCV with radiographic 
progression, either quantitatively or qualitatively, so unfortunately we were unable to extract data to perform a meta-analysis. More severe radiographic progression, specified by the changes in the Larsen score compared to the baseline, was observed in anti-MCV positive patients than in anti-MCV negative patients in the subgroup of early RA patients [30,37]. Although no significant correlation of anti-MCV with the Larsen score or the number of erosions of joints on ultrasonography in patients with established RA was observed in the studies included here, the anti-MCV titer was suggested as a marker of disease activity [12, 15, 27, 31]. Long-term prospective randomized controlled trials are needed to determine the predictive value of baseline anti-MCV for RA progression.

To date, there are two authoritative criteria for the diagnosis of RA: the 2010 ACR/EULAR criteria aimed at classifying early RA and demonstrating higher sensitivity but lower specificity, especially in patients aged over 60 years old compared to the 1987 ACR criteria, while the latter was considered to predict a more erosive disease [14]. However, existing data suggested that not fulfilling the 2010 ACR/EULAR criteria does not rule out RA diagnosis, for example, those seronegative for RF or anti-CCP RA patients with less than 10 involved joints [48]. $R F$ is the only biomarker in the ACR 1987 criteria, while the ACR/ EULAR 2010 criteria use both RF and ACPA, which is typically tested as anti-CCP antibodies [5, 49, 50]. Neither of these two criteria mentioned anti-MCV as a new valid diagnostic marker. Additionally, anti-carbamylated protein (anti-CarP) IgA antibodies were observed as another distinct autoantibody in the sera of RA patients in 2011, and is regarded as a promising biomarker for detecting early RA and very early RA $[43,51]$. A sensitivity of $42 \%$ and an overall specificity of $89 \%$ of antiCarP antibodies for RA were reported, and the correlation between antiCarP antibodies and disease activity is still debated [43, 52, 53]. Nevertheless, no study included in this meta-analysis mentioned the performance of anti-CarP in the diagnosis for RA.

The early identification of RA is a great challenge, as the prognosis would be significantly improved with early diagnosis and intervention of RA. It is implied that anti-MCV may behave a bit differently in the diagnosis of early RA compared with established RA [4]. Meanwhile, interpreting diagnostic values of the combined markers might be the solution for detecting serum negative RA, but a tradeoff exists between sensitivity and specificity. Therefore, more diagnostic trials of the combined performance of anti-MCV and anti-CCP for RA diagnosis and prognosis, especially the combined specificity, as well as its application in the diagnosis of early RA compared with established RA, are required.

In summary, anti-MCV demonstrates a comparable diagnostic value to anti-CCP and RF, therefore is suggested as an alternative biomarker for RA classification in clinical practice, and may be written into the next authoritative criteria.

Acknowledgments: We thank Professor Yun-Xian Yu from School of Public Health, Zhejiang University who provided expertise that greatly assisted our research. We thank Chief Physician Jing Xue for comments that greatly improved the manuscript.

Author contributions: J-N. Zhu, L-Y. Nie and X-Y. Lu had full access to all of the data in the study and take responsibility for the integrity of the data and the accuracy of the data analysis, they contributed equally to this work. Concept and design: J-N. Zhu, L-Y. Nie, X-Y. Lu. Acquisition, analysis, or interpretation of data: All authors. Drafting of the manuscript: J-N. Zhu, L-Y. Nie, X-Y. Lu. Critical revision of the manuscript for important intellectual content: All authors. Statistical analysis: J-N. Zhu, L-Y. Nie. Administrative, technical, or material support: All authors. Supervision: X-Y. Lu, H-X. Wu. All the authors have accepted responsibility for the entire content of this submitted manuscript and approved submission.

Research funding: None declared.

Employment or leadership: None declared.

Honorarium: None declared.

Competing interests: The funding organization(s) played no role in the study design; in the collection, analysis, and interpretation of data; in the writing of the report; or in the decision to submit the report for publication.

\section{References}

1. Smolen JS, Aletaha D, McInnes IB. Rheumatoid arthritis. Lancet 2016;388:2023-38.

2. Laiho K, Tuomilehto J, Tilvis R. Prevalence of rheumatoid arthritis and musculoskeletal diseases in the elderly population. Rheumatol Int 2001;20:85-7.

3. Mok CC. Morning stiffness in elderly patients with rheumatoid arthritis: what is known about the effect of biological and targeted agents? Drugs Aging 2018;35:477-83.

4. Pruijn GJ, Wiik A, van Venrooij WJ. The use of citrullinated peptides and proteins for the diagnosis of rheumatoid arthritis. Arthritis Res Ther 2010;12:203.

5. Gavrila BI, Ciofu C, Stoica V. Biomarkers in rheumatoid arthritis, what is new? J Med Life 2016;9:144-8.

6. Luime JJ, Colin EM, Hazes JM, Lubberts E. Does anti-mutated citrullinated vimentin have additional value as a serological 
marker in the diagnostic and prognostic investigation of patients with rheumatoid arthritis? A systematic review. Ann Rheum Dis 2010;69:337-44.

7. Lee YH, Bae SC, Song GG. Diagnostic accuracy of anti-MCV and anti-CCP antibodies in rheumatoid arthritis: a meta-analysis. $Z$ Rheumatol 2015;74:911-8.

8. Qin X, Deng Y, Xu J, Li TJ, Li S, Zhao JM. Meta-analysis: diagnostic value of serum anti-mutated citrullinated vimentin antibodies in patients with rheumatoid arthritis. Rheumatol Int 2011;31:785-94.

9. Zahran WE, Mahmoud MI, Shalaby KA, Abbas MH. Unique correlation between mutated citrullinated vimentine IgG autoantibodies and markers of systemic inflammation in rheumatoid arthritis patients. Indian J Clin Biochem 2013;28:272-6.

10. Yousefghahari B, Alhooei S, Soleimani-Amiri MJ, Guran A. Comparison of sensitivity and specificity of anti-CCP and anti-MCV antibodies in an Iranian cohort of patients with rheumatoid arthritis. Caspian J Intern Med 2013;4:702-6.

11. Damjanovska L, Thabet MM, Levarth EW, Stoeken-Rijsbergen G, van der Voort El, Toes RE, et al. Diagnostic value of anti-MCV antibodies in differentiating early inflammatory arthritis. Ann Rheum Dis 2010;69:730-2.

12. Iwaszkiewicz C, Puszczewicz M, Bialkowska-Puszczewicz G. Diagnostic value of the anti-Sa antibody compared with the anticyclic citrullinated peptide antibody in rheumatoid arthritis. Int J Rheum Dis 2015;18:46-51.

13. Nicaise Roland P, Grootenboer Mignot S, Bruns A, Hurtado M, Palazzo E, Hayem G, et al. Antibodies to mutated citrullinated vimentin for diagnosing rheumatoid arthritis in anti-CCP-negative patients and for monitoring infliximab therapy. Arthritis Res Ther 2008;10:R142.

14. Berglin E, Dahlqvist SR. Comparison of the 1987 ACR and 2010 ACR/EULAR classification criteria for rheumatoid arthritis in clinical practice: a prospective cohort study. Scand J Rheumatol 2013;42:362-8.

15. Liu X, Jia R, Zhao J, Li Z. The role of anti-mutated citrullinated vimentin antibodies in the diagnosis of early rheumatoid arthritis. J Rheumatol 2009;36:1136-42.

16. Nicaise-Roland P, Nogueira L, Demattei C, de Chaisemartin L, Rincheval N, Cornillet M, et al. Autoantibodies to citrullinated fibrinogen compared with anti-MCV and anti-CCP2 antibodies in diagnosing rheumatoid arthritis at an early stage: data from the French ESPOIR cohort. Ann Rheum Dis 2013;72:357-62.

17. Bizzaro N, Tonutti E, Tozzoli R, Villalta D. Analytical and diagnostic characteristics of 11 2nd- and 3rd-generation immunoenzymatic methods for the detection of antibodies to citrullinated proteins. Clin Chem 2007;53:1527-33.

18. Kim SK, Bae J, Lee H, Kim JH, Park SH, Choe JY. Greater prevalence of seropositivity for anti-cyclic citrullinated peptide antibody in unaffected first-degree relatives in multicase rheumatoid arthritis-affected families. Korean J Intern Med 2013;28:45-53.

19. Maraina CH, Nurdayana AK, Rusni D, Azwany Y. Diagnostic value of anti-modified citrullinated vimentin in rheumatoid arthritis. Int J Rheum Dis 2010;13:335-9.

20. Kaptanoglu E, Nadir I, Bakici Z, Hayta E, Turkmen M, Sezer H, et al. Differentiation of rheumatoid arthritis from HCV infection: rheumatoid factor, anti-cyclic citrullinated peptide or anti-mutated citrullinated vimentin? Turk Romatoloji Dergisi 2010;25:19-23.
21. Mutlu N, Bicakcigil M, Tasan DA, Kaya A, Yavuz S, Ozden Al. Comparative performance analysis of 4 different anti-citrullinated protein assays in the diagnosis of rheumatoid arthritis. J Rheumatol 2009;36:491-500.

22. Diaz-Toscano ML, Olivas-Flores EM, Zavaleta-Muniz SA, GamezNava JI, Cardona-Munoz EG, Ponce-Guarneros M, et al. Comparison of two assays to determine anti-citrullinated peptide antibodies in rheumatoid arthritis in relation to other chronic inflammatory rheumatic diseases: assaying anti-modified citrullinated vimentin antibodies adds value to second-generation anti-citrullinated cyclic peptides testing. Biomed Res Int 2014;2014:198198.

23. Zhu T, Feng L. Comparison of anti-mutated citrullinated vimentin, anti-cyclic citrullinated peptides, anti-glucose-6-phosphate isomerase and anti-keratin antibodies and rheumatoid factor in the diagnosis of rheumatoid arthritis in Chinese patients. Int J Rheum Dis 2013;16:157-61.

24. Svard A, Kastbom A, Soderlin MK, Reckner-Olsson A, Skogh T. A comparison between IgG- and IgA-class antibodies to cyclic citrullinated peptides and to modified citrullinated vimentin in early rheumatoid arthritis and very early arthritis. J Rheumatol 2011;38:1265-72.

25. Soos L, Szekanecz Z, Szabo Z, Fekete A, Zeher M, Horvath IF, et al. Clinical evaluation of anti-mutated citrullinated vimentin by ELISA in rheumatoid arthritis. J Rheumatol 2007;34:1658-63.

26. Dejaco C, Klotz W, Larcher H, Duftner C, Schirmer M, Herold M. Diagnostic value of antibodies against a modified citrullinated vimentin in rheumatoid arthritis. Arthritis Res Ther 2006;8:R119.

27. Wagner E, Skoumal M, Bayer PM, Klaushofer K. Antibody against mutated citrullinated vimentin: a new sensitive marker in the diagnosis of rheumatoid arthritis. Rheumatol Int 2009;29: 1315-21.

28. Zehairy M, Soliman E, Daghaidy A. Antibodies to mutated citrullinated vimentin in patients with chronic hepatitis $C$ virus genotype IV infection-related arthropathy. Rheumatol Int 2012;32:3495-501.

29. Bang H, Egerer K, Gauliard A, Luthke K, Rudolph PE, Fredenhagen $\mathrm{G}$, et al. Mutation and citrullination modifies vimentin to a novel autoantigen for rheumatoid arthritis. Arthritis Rheum 2007;56:2503-11.

30. Barouta G, Katsiari CG, Alexiou I, Liaskos C, Varna A, Bogdanos DP, et al. Anti-MCV antibodies predict radiographic progression in Greek patients with very early ( $<3$ months duration) rheumatoid arthritis. Clin Rheumatol 2017;36:885-94.

31. Sizova L. Diagnostic value of antibodies to modified citrullinated vimentin in early rheumatoid arthritis. Hum Immunol 2012;73:389-92.

32. Lima I, Oliveira RC, Atta A, Marchi S, Barbosa L, Reis E, et al. Antibodies to citrullinated peptides in tuberculosis. Clin Rheumatol 2013;32:685-7.

33. Sghiri R, Bouajina E, Bargaoui D, Harzallah L, Fredj HB, Sammoud S, et al. Value of anti-mutated citrullinated vimentin antibodies in diagnosing rheumatoid arthritis. Rheumatol Int 2008;29:59-62.

34. Sahin O, Kaptanoglu E, Bakici MZ, Sezer H, Elden H, Hizmetli S. Diagnostic value of autoantibodies against citrullinated peptide antigens in rheumatoid arthritis: comparison of different commercial kits. Turk J Rheumatol 2011;26:13-8.

35. Reyes-Castillo Z, Palafox-Sanchez CA, Parra-Rojas I, MartinezBonilla GE, del Toro-Arreola S, Ramirez-Duenas MG, et al. 
Comparative analysis of autoantibodies targeting peptidylarginine deiminase type 4, mutated citrullinated vimentin and cyclic citrullinated peptides in rheumatoid arthritis: associations with cytokine profiles, clinical and genetic features. Clin Exp Immunol 2015;182:119-31.

36. Renger F, Bang H, Feist E, Fredenhagen G, Natusch A, Backhaus $M$, et al. Immediate determination of ACPA and rheumatoid factor - a novel point of care test for detection of anti-MCV antibodies and rheumatoid factor using a lateral-flow immunoassay. Arthritis Res Ther 2010;12:R120.

37. Mathsson L, Mullazehi M, Wick MC, Sjoberg O, van Vollenhoven $\mathrm{R}$, Klareskog $\mathrm{L}$, et al. Antibodies against citrullinated vimentin in rheumatoid arthritis: higher sensitivity and extended prognostic value concerning future radiographic progression as compared with antibodies against cyclic citrullinated peptides. Arthritis Rheum 2008;58:36-45.

38. El-Barbary AM, Kassem EM, El-Sergany MA, Essa SA, Eltomey MA. Association of anti-modified citrullinated vimentin with subclinical atherosclerosis in early rheumatoid arthritis compared with anti-cyclic citrullinated peptide. J Rheumatol 2011;38:828-34.

39. Bartoloni E, Alunno A, Bistoni O, Bizzaro N, Morozzi G, Doria $A$, et al. Diagnostic value of anti-mutated citrullinated vimentin in comparison to anti-cyclic citrullinated peptide and anti-viral citrullinated peptide 2 antibodies in rheumatoid arthritis: a multicentric study by firma group. Ann Rheum Dis 2013;71:607.

40. Al-Shukaili A, Al-Ghafri S, Al-Marhoobi S, Alkaabi J. Evaluation of anti-mutated citrullinated vimentin antibodies, anticyclic citrullinated Peptide antibodies and rheumatoid factor in omani patients with rheumatoid arthritis. Int J Rheumatol 2012;2012:285-854.

41. Meyer PW, Ally MT, Hodkinson B, Anderson R, Tikly M. Comparison of the diagnostic potential of three anti-citrullinated protein antibodies as adjuncts to rheumatoid factor and CCP in a cohort of South African rheumatoid arthritis patients. Rheumatol Int 2018;38:993-1001.

42. Besada E, Nikolaisen C, Nossent H. Diagnostic value of antibodies against mutated citrullinated vimentin for rheumatoid arthritis. Clin Exp Rheumatol 2011;29:85-8.

43. Trouw LA, Rispens T, Toes RE. Beyond citrullination: other posttranslational protein modifications in rheumatoid arthritis. Nat Rev Rheumatol 2017;13:331-9.

44. Kuna AT. Mutated citrullinated vimentin antibodies in rheumatoid arthritis. Clin Chim Acta 2012;413:66-73.
45. Gonzalez-Lopez L, Rocha-Munoz AD, Ponce-Guarneros M, Flores-Chavez A, Salazar-Paramo M, Nava A, et al. Anti-cyclic citrullinated peptide (anti-CCP) and anti-mutated citrullinated vimentin (anti-MCV) relation with extra-articular manifestations in rheumatoid arthritis. J Immunol Res 2014;2014:536050.

46. Harre U, Georgess D, Bang H, Bozec A, Axmann R, Ossipova E, et al. Induction of osteoclastogenesis and bone loss by human autoantibodies against citrullinated vimentin. J Clin Invest 2012;122:1791-802.

47. Engdahl C, Bang H, Dietel K, Lang SC, Harre U, Schett G. Periarticular bone loss in arthritis is induced by autoantibodies against citrullinated vimentin. J Bone Miner Res 2017;32:1681-91.

48. Jung SJ, Lee SW, Ha YJ, Lee KH, Kang Y, Park MC, et al. Patients with early arthritis who fulfil the 1987 ACR classification criteria for rheumatoid arthritis but not the 2010 ACR/EULAR criteria. Ann Rheum Dis 2012;71:1097-8.

49. Funovits J, Aletaha D, Bykerk V, Combe B, Dougados M, Emery P, et al. The 2010 American College of Rheumatology/European League Against Rheumatism classification criteria for rheumatoid arthritis: methodological report phase I. Ann Rheum Dis 2010;69:1589-95.

50. Aletaha D, Neogi T, Silman AJ, Funovits J, Felson DT, Bingham 3rd CO, et al. 2010 Rheumatoid arthritis classification criteria: an American College of Rheumatology/European League Against Rheumatism collaborative initiative. Arthritis Rheum 2010;62:2569-81.

51. Shi J, Knevel R, Suwannalai P, van der Linden MP, Janssen GM, van Veelen PA, et al. Autoantibodies recognizing carbamylated proteins are present in sera of patients with rheumatoid arthritis and predict joint damage. Proc Natl Acad Sci USA 2011;108:17372-7.

52. Kaneko C, Kobayashi T, Ito S, Sugita N, Murasawa A, Nakazono $\mathrm{K}$, et al. Circulating levels of carbamylated protein and neutrophil extracellular traps are associated with periodontitis severity in patients with rheumatoid arthritis: a pilot case-control study. PLoS One 2018;13:e0192365.

53. Johansson L, Arlestig L, Kokkonen H, Brink M, RantapaaDahlqvist S. An increased concentration of receptor activator of nuclear factor kappa-B ligand pre-dates the onset of rheumatoid arthritis. Rheumatology (Oxford) 2017;56:2190-6.

Supplementary Material: The online version of this article offers supplementary material (https://doi.org/10.1515/cclm-2019-0167). 